\title{
Mitochondrial alterations may underlie race-specific differences in cancer risk and outcome
}

\author{
Jennifer L. Beebe-Dimmer' and Kathleen A. Cooney² \\ 'Barbara Ann Karmanos Cancer Institute, Population Studies and Disparities Research, Wayne State University, Detroit, Michigan, USA. Department of Medicine, Duke Cancer Institute, Duke University, \\ Durham, North Carolina, USA.
}

\begin{abstract}
African Americans are at increased risk of cancer and associated mortalities compared with European American populations. Socioeconomic, cultural, and biological factors have been implicated in this discrepancy. In this issue of the $J C I$, Piyarathna et al. identify a set of genes that are upregulated in a number of tumor types in African American cancer patients as compared with European American patients. These genes were associated with enhanced oxidative phosphorylation and upregulation of transcription factors that promote mitochondrial biogenesis, resulting in greater numbers of mitochondria in tumor samples from African American subjects. Together, these results indicate that mitochondria dysfunction may underlie the increased cancer incidence and poor outcomes observed in African American patients.
\end{abstract}

tion factors within the OXPHOS pathway in tumors from African American patients. Because these two genes act in concert to regulate mitochondrial biogenesis, Piyarathna et al. hypothesized that mitochondrial content would be higher in tumors from African American subjects compared with tumors derived from patients of European American descent. This hypothesis was supported in an experiment using tissue microarrays constructed from prostate, laryngeal, and oral cancer specimens.

The potential impact of the findings of Piyarathna and colleagues is considerable, not only in furthering our knowledge of the biological basis of cancer health disparities, but in moving the needle with respect to personalized medicine. Primarily responsible for cellular respiration and energy production, mitochondria have been shown to play an important role in oxidative stress, cell signaling, and cell death; therefore, it is logical to conclude that these organelles may be important in carcinogenesis (4). A better understanding of the role of mitochondria and the impact of their dysfunction in cancer initiation and progression will be critical in developing the next generation of cancer therapeutics and for exploiting the full potential of existing mitochondrial inhibitors, such as metformin and phenformin (5).

Moreover, the work of Piyarathna et al. contributes to a growing body of literature that supports a role for mitochondria in cancer health disparities. Prior studies have been mixed and focused on specific cancers. For example, previous research in prostate cancer found that mitochondrial content measured by immunohistochemistry positively correlated with clinical characteristics associated with aggressive disease, including pathologic stage, Gleason score, and early evidence of prostate-specific antigen recurrence following treatment (6). This study was conducted in a large European cohort, so the potential
Conflict of interest: JLBD is a consultant for The CUSP Group, LLC., a uro-oncology trials management organization. KAC is coinventor on a patent (no. 9,593,380) related to the discovery of HOXB13 as a prostate cancer susceptibility gene. Copyright: () 2019, American Society for Clinical Investigation.

Reference information: / Clin Invest. 2019;129(6):2187-2188. https://doi.org/10.1172/JCI128707. 
association of mitochondrial content and race could not be addressed. Koochekpour et al. (7) observed mitochondrial DNA depletion in prostate tissue from African American patients and suggested that lower mitochondrial DNA content contributes to differences by race in prostate cancer incidence and progression. Additional research has focused on both germline and somatic mutations in mitochondrial DNA in prostate cancer and other malignancies with variable findings (8). Finally, use of metformin as a prostate cancer chemopreventive has shown some promise, as it has been linked to reduced risk of recurrence, metastatic disease, and death in men with concomitant type 2 diabetes (9). However, whether or not there are differences by race and/or ethnicity in sensitivity to drugs such as metformin requires further study.

\section{Future considerations and conclusions}

While the findings of Piyarathna et al. are intriguing, there are some points that merit further consideration. The tumor types used for the initial discovery work were selected because at least ten African American patients were enrolled in TCGA for that particular cancer. Race was obtained by self-report, and the number of African American cases (overall 12.7\% in TCGA) compared with European American cases was likely small. In addition, individuals typically report their race based on skin color rather than ancestry, thereby creating an opportunity for misclassification (10). Furthermore, African Americans can have differing amounts of African and
European ancestry, and we concur with the recommendation by Piyarathna et al. that future studies focus on use of ancestryinformative genetic markers to more accurately classify individuals in studies of biological contributions to cancer disparities. Additionally, the overall number of cancer cases in the Piyarathna et al. report is small and the enrichment of OXPHOS appears to be variable across tumor types and should be further explored. Finally, the potential role(s) of mitochondria in cancer incidence and aggressiveness are complex; therefore, mitochondrial function, in addition to total number of these organelles, merits investigation.

In summary, studies that focus simultaneously on multiple cancer types may help identify signals that will lead to recognition of common biological mechanisms that contribute to increased cancer incidence and/or aggressiveness in African American individuals. For example, while PGC1 $\alpha$ has been extensively studied in cancer (11), to our knowledge this is the first report of its potential contribution to cancer disparities. Future studies of OXPHOS-associated genes in wellannotated clinical series of racially diverse patients characterized with ancestry-informative markers are warranted.

Address correspondence to: Jennifer L. Beebe-Dimmer, Wayne State University, Barbara Ann Karmanos Cancer Institute, Program Leader Population Studies and Disparities Research, 4100 John R, Detroit, Michigan, 48201, USA. Phone: 313.578.4209; Email:dimmerj@karmanos. org. Or to: Kathleen A. Cooney, Duke University School of Medicine, 2301 Erwin Road, Suite 1102, Durham, North Carolina, 27710, USA. Phone: 919.681.2452; Email: kathleen.cooney@duke.edu.

1. Goding Sauer A, Siegel RL, Jemal A, Fedewa SA. Current prevalence of major cancer risk factors and screening test use in the United States: disparities by education and race/ethnicity. Cancer Epidemiol Biomarkers Prev. 2019;28(4):629-642.

2. Davis MB, Newman LA. Breast cancer disparities: how can we leverage genomics to improve outcomes? Surg Oncol Clin N Am. 2018;27(1):217-234.

3. Piyarathna DWB, et al. ERR1- and PGC1 $\alpha$-associated mitochondrial alterations correlate with pan-cancer disparity in African Americans. JClin Invest. 2019;129(6):2351-2356.

4. Vyas S, Zaganjor E, Haigis MC. Mitochondria and cancer. Cell. 2016;166(3):555-566.

5. Weinberg SE, Chandel NS. Targeting mitochondria metabolism for cancer therapy. Nat Chem Biol. 2015;11(1):9-15.

6. Grupp K, et al. High mitochondria content is associated with prostate cancer disease progression. Mol Cancer. 2013;12(1):145.

7. Koochekpour S, Marlowe T, Singh KK, Attwood $\mathrm{K}$, Chandra D. Reduced mitochondrial DNA content associates with poor prognosis of prostate cancer in African American men. PLOS ONE. 2013;8(9):e74688.

8. Choudhury AR, Singh KK. Mitochondrial determinants of cancer health disparities. Semin Cancer Biol. 2017;47:125-146.

9. Chong RW, Vasudevan V, Zuber J, Solomon SS. Metformin has a positive therapeutic effect on prostate cancer in patients with type 2 diabetes mellitus. Am J Med Sci. 2016;351(4):416-419.

10. Mersha TB, Abebe T. Self-reported race/ ethnicity in the age of genomic research: its potential impact on understanding health disparities. Hum Genomics. 2015;9:1.

11. Tan Z, et al. The role of PGC1 $\alpha$ in cancer metabolism and its therapeutic implications. Mol Cancer Ther. 2016;15(5):774-782. 